\title{
High School Students' Language Related Struggles with Contextualized Differential Equations
}

\author{
Zakaria Ndemo \\ Department of Science and Mathematics Education, Faculty of Science Education, Bindura University of Science Education, Bindura, \\ Zimbabwe
}

\section{Email address: \\ zndemo@gmail.com}

\section{To cite this article:}

Zakaria Ndemo. High School Students' Language Related Struggles with Contextualized Differential Equations. International Journal of Applied Mathematics and Theoretical Physics. Vol. 5, No. 1, 2019, pp. 20-31. doi: 10.11648/j.ijamtp.20190501.13

Received: January 10, 2019; Accepted: March 15, 2019; Published: April 18, 2019

\begin{abstract}
Upon observing with concern that the majority of high school students experienced severe language related difficulties when solving contextualized differential equations, the researcher then decided to investigate the kinds of such challenges and their impact on students' learning of differential equations. A sample of 10 mathematics students was selected from one urban high school in one province in Zimbabwe. Written tasks and follow up interviews were employed as data collection tools for the study. Content analysis technique was applied to the written responses and interview transcriptions to obtain a revealing picture of how the kinds of the language related challenges interfere with the growth of mathematical content. The study revealed that the students struggled with interpreting and formulating differential equations from given mathematical situations. Further, interpretation of given initial conditions posed a challenge to the learners. A major consequence of these language related challenges was that the students could not generate complete solutions and lack of interplay between conceptual and procedural fluency was one of the insights generated from this study with regards to the learning of differential equations. The study findings have important implications for instruction in high school mathematics lessons such as the need to develop and foster the students' abilities to engage in adaptive reasoning and use multiple modes of presenting content in order to promote students' understanding of contextualized differential equations.
\end{abstract}

Keywords: Differential Equations, Contextualized Mathematics. High School Mathematics, Language Barriers

\section{The Research Problem}

\subsection{Motivation and Context of the Study}

Reccomini et al. [1] have underscored the important role played by language in mathematics learning so much such that the attention of many mathematics educators need to focus on content-specific vocabulary of mathematics. The call for increased attention that should be paid to language matters in mathematics education stems from an understanding that mathematics is a sense making process in which students should engage with mathematics language through reading, writing and even speaking as suggested by Lemke [2]. Van Rinsveld [3] writes that the ability by students to solve word problems depends on their command of the language of instruction. In other words, learning of word problems is constrained or unlocked by the learner's command of the language of instruction. Sarabi and Abdul Gafoor [4] share the view that language is an important factor because it allows students to describe generalizations, patterns and justify mathematical propositions. The concept of a differential equation is a crucial requisite for solving a variety of problems in applied mathematics, physics and economics. Many fundamental laws of physics and chemistry are formed using the concept of a differential equation. The solution of a problem on a differential equation relies on key elements such as rigor, logic, creativity, and interplay between procedural and conceptual fluency which are supported by language. Hence, a student's language proficiency is a key determining factor in modeling of phenomena using differential equations.

In spite of the crucial role played by language in developing the concept of a differential equation the author of this article observed that most students were unable to understand the language and vocabulary of contextualized differential equations. During several years of teaching high school mathematics the researcher observed that learners were unable 
to understand and relate to the context of the problem and in some instances they found it difficult to identify basic related ideas that form the gist of the problem solving process. These observations have also been confirmed by literature. For instance, Cummis et al. [5] have reported that learners have difficulties in processing and resolving contextualized mathematical tasks. According to Martiniello [6] students' struggles with the language of mathematics can be attributed to the nature of mathematics discourse. Furthermore, Martiniello [6] writes that mathematics involves use of symbolism and content specific terminology that could be overwhelming for the student. In light of students' discomforts in learning differential equations, it is the intent of this study to describe and explain the nature of language related challenges faced when students solve tasks on contextualized differential equations and how such challenges affect the development of the concept of a differential equation among Advanced level students. Hence, the overarching question that guided the study is now stated in the next section.

\subsection{Research Question and Objectives}

How do language related challenges impede the growth of the concept of a differential equation among high school mathematics students?

By describing and explaining how language related matters that characterize students' attempts to comprehend contextualized differential equations impede on the growth of students' knowledge of differential equations, the study seeks to account for the students' problem solving behavior. Further, the intent of the study is to explain how such language connected students' discomforts with differential equations interfere with the growth of mathematics knowledge. These accomplishments are, in the researcher's view, important subject content knowledge (SCK) considerations. Lesseig [7] describes SCK as knowledge of students' typical conceptions and misconceptions of a specific content area.

\section{Theoretical Considerations}

\subsection{Contextualized Differential Equations}

Clarke and Helme [8] cited by Ndemo and Mtetwa [9] define context as the real situation in which a mathematical task is embedded. Clarke and Helme [8] call this real situation the objective figurative context. Ndemo and Mtetwa [9] write that when the teacher and the students attempt to draw meaning from the objective figurative we obtain what is known as the social or interactive context. Finally, an individual's construal of the objective figurative context is called the subjective or personalized context. We now use an example drawn from the mathematics content area of differential equations to illustrate the notion of context just described here.

Example 1: Contextualized differential equations

A girl returning from the milling point is carrying mealie-meal in a cylindrical container. The container has a hole at its base and mealie-meal trickles out through this hole.
It is estimated that the rate of reduction of mealie-meal is proportional to the mass $\mathrm{m}$ of mealie-meal remaining in the container, so that this situation can be modeled by the differential equation

$\frac{d m}{d t}=-\frac{k}{5} m$, where $\mathrm{k}$ is a constant.

i. Find the general solution of this differential equation and show that it reduces to

ii. $m=m_{0} e^{-\frac{k t}{5}}$ where $m_{0}$ is the initial mass of the mealie-meal.

iii. The girl takes 2 hours to walk from the milling point to her home. Given that after one hour, ten percent of the mealie meal is lost,

iv. Calculate the percentage of mealie-meal in the container when she arrives home. Sketch a graph showing the variation of the mass of the mealie-meal during the two-hour journey.

The social context refers to the teacher and students' interpretation of the situation in which the mathematics ideas are embedded, for instance the rate of decrease of mass of mealie-meal, $\left(-\frac{d m}{d t}\right)$ captured by the term "mealie-meal trickles out". The social interactive context described here can be differentiated from the personalized figurative context which consists of each individual student's interpretation of the problem situation. The focus of the study was on students' personalized figurative contexts of the contextualized tasks on differential equations. Contextualized mathematical tasks illustrated here can be different from context free or decontextualized mathematical tasks which we now exemplify.

Example 2: Decontextualized task on differential equations Solve the equation, $\frac{y}{x\left(x^{2}+3\right)} \frac{d y}{d x}=e^{-y}$, given that $y=$ 1 when $x=0$.

Hence, example 2 reveals the fact that a context free mathematical task is one in which the mathematical ideas involved are not embedded in a real life situation.

\subsection{Related Studies}

Lager [10] reports that students experience severe difficulties in extracting meaning from narrative descriptions of the problem situation. Kilpatrick et al. [11] write that students' difficulties with contextualized mathematical tasks can be overcome when the students develop adaptive reasoning in order to make sense of the objective figurative contexts of mathematical tasks. Kilpatrick et al. [11] define adaptive reasoning as one that involves the ability by students to think logically about the relations among concepts involved in the task at hand. The argument is that students can only be successful problem solvers if they develop a deep understanding of the technical language of contextualized differential equations. Such an understanding may call for identifying initial conditions involved in the differential equation, representing the narrative form in symbolic form. The intent of the study is to describe and explain the language barriers illuminated in students' efforts to resolve contextualized ordinary differential equations.

Boulet [12] suggests that language comprehension deficits 
may inhibit the conceptual understanding of the problem because the conceptual and procedural fluency of the learner are constrained by language. An illustration of the influence of language in problem solving can be drawn from Selden and Selden's [13] description of the distinction between synthetic and analytic definitions in mathematics. Mathematical discourse is constituted by a number of words and terms with different analytic and synthetic meanings. For example, every day or synthetic meaning of the word order is differentiated from its analytic use in differential equations. In the synthetic sense, the word order describes an arrangement, a command by a superior while in the topic on differential equations order denotes whether the differential equation involves first or second or even higher derivatives of the variables. The argument is that students' struggles with mathematics can be caused by failure to differentiate between synthetic and analytic meanings of mathematical terms.

There is an intimate relationship between students' knowledge of concepts and the words they use to talk about those concepts. Hence, language is at the heart of mathematical activity. Mulwa [14] asserts that language is a powerful instrument in the formation of concepts and development of particular abilities. As such language facilitates the thinking process which is essential for the discovery of new mathematical ideas. Reccomini et al. [1] define language as the words, their pronunciation and the methods of combining them used and understood by a community. The manner in which language is used is called vocabulary. In the context of mathematics learning vocabulary is used to denote a learner's ability to use words to explain, justify and communicate an individual's understanding of a mathematical concept. Hence, a student's grasp of mathematical vocabulary is essential as it affords the student access to mathematical concepts.

The distinction between synthetic and analytic meanings of mathematical concepts is strikingly similar to the distinction between academic and everyday language. Zwiers [15] defines academic language as the set of words and phrases that describe content area knowledge and procedures, express thinking processes about concepts involved so as to create cohesion and clarity in the mathematical discourse. This definition reveals that competence in academic language is not only limited to the acquisition of content vocabulary but it encompasses ways of thinking and acting using words and phrases that constitute a mathematical discourse. The acquisition of academic language is confined to the classroom. Consequently classroom discourse patterns and activities both can develop and impede language growth. On the other hand, everyday language is non-technical and may include words spoken in informal conversations at home and other social settings.

During learning students are expected to draw on multiple semiotic systems in order to construct knowledge. Semiotic systems are so called because within those systems symbols, written language and visual representations such as graphs are employed to make and share meanings. Use of visual representations such as graphs in semiotic systems of creating knowledge helps learners to unpack and repack meanings in problem contexts. Furthermore, Hewitt-Bradshaw [16] suggests that students must learn to understand and use different semiotic codes to translate their knowledge into multiple modes of representation, an exercise that would in turn strengthen their mathematical knowledge. However, a study by Hewitt-Bradshaw [16] revealed that students had difficulties in creating, interpreting and reasoning with visual representations such as graphs. Hewitt-Brandshaw's finding justifies the need for further investigation into classroom discourse in content areas to evaluate the extent to which language competence is a factor in students' learning of differential equation.

Hewitt-Bradshaw [15] and Reccomini et al. [1] have found that students often develop surface-level understanding of mathematics concepts and hence would not gain the desired deep understanding. Such superficial understanding of mathematics is manifested through for instance, students being not able to distinguish between verb-preposition collocations such as increased/decreased by and increased/decreased to when solving tasks on differential equations. Fatimanssa and Kushandi [17] have noted that solving contextualized mathematical ideas requires complex procedural steps and a good command of structural relationship of mathematical ideas of the task at hand. Further, Gagnon and Abel [18] have argued that subtle and abstract features such as differences in vocabulary and terms denoting relationships among ideas make the learning of mathematics difficult. Hence, students' failure to differentiate between the two forms of verb-preposition collocations can impede efforts to unpack meanings expressed in problem contexts and reorganize such meanings in ways that make sense to the students. Further, learning of mathematics has been characterized by lack of grasp by learners of the distinction between mathematical notation and symbols and the ideas embedded in those notations. Yet all too often greater emphasis has been on notation rather than the mathematics conveyed by those symbols. For instance, learners have referred to the fraction $\frac{2}{3}$ as 2 over 3 instead of 2 out of 3 . In other words, the learners' focus has been on how the fraction is written symbolically as opposed to ideas expressed by the fraction. The researcher argues here that addressing notation rather than the ideas captured by the notation/symbols renders mathematical discourse ineffective.

The foregoing discussion has shown that language is at the heart of mathematical activity. van Rinsveld et al. [3] emphasize the importance of language of instruction in mathematics by explaining that students with a weak command of language are more likely to experience difficulties in resolving word problems. Hence, a good command of the language of differential equations is crucial. This is so because by articulating the principles, the concepts and rationale behind steps of the problem solving process, a learner has an opportunity to reinforce, and deepen his or her understanding of knowledge structures of given mathematics content as suggested by Huang et al. [19]. Developing a profound understanding of students' language related difficulties would then allow the instructors to build an awareness of and become 
more sensitive to language related challenges which obturate concept development as suggested by Boulet [12]. Hence, this study examined students' interpretation of language and symbols used in learning differential equations. The underlying theoretical principle is that language is an instrument of thought that determines the kinds of students' construal of contextualized differential equations.

\section{Methods}

\subsection{Research Design}

Durrheiim et al. [20] define a research design as a strategic framework for action that serves as a bridge between research questions and the implementation of the research strategy. Durrheiim et al. [20] argue that a good and sound research design will ensure that the information obtained is relevant to the research questions and should provide results that are judged to be credible. In this study a survey design was considered to be appropriate for the purpose of exploring students' language related challenges with the learning of contextualized differential equations at Advanced level. A cross-sectional survey was employed to uncover the kinds of challenges held by the students and to determine how such challenges hinder students' learning of differential equations.

\subsection{Population and Sampling}

The author of this article sought expertise assistance of Advanced level mathematics teachers to divide students into three groups basing on those teachers' knowledge of the students' academic performance. The groups included high performers, middle performers and low performers. Simple random sampling was employed to each of the three groups to select a sample of size 3 from the high performers' group, 3 students from low performers and 4 from the middle performers' group bringing the sample size to 10 . The researcher clarifies that the intent of the study was to generate insights about students' thoughts as they engaged with tasks on differential equations. In other words, the goal was developing an understanding of students' thinking processes and account for why students tackled the tasks in the manner they did. Hence, a small sample was used because focus was not on generalizability of findings. Simple random sampling was used to ensure that students of all abilities had equal chances of being included in the sample. Hence, the cross sectional survey design employed in this study had a qualitative orientation despite use of the simple random sampling technique usually associated with quantitative designs.

\subsection{Research Instruments}

To generate data for this qualitative investigation, the researcher prepared a task sheet comprising two tasks. The task sheet contained two contextualized tasks on differential equations. The intent of the tasks was to tease out language related challenges encountered by Advanced level students when they were solving differential equations. Follow up interviews were conducted and audio-recorded and later on transcribed. Follow up interviews were done with selected students whose answers were not clear and required clarification. Follow up interviews were also done with students who had experienced some impasses during their solution attempts. The researcher was "keen to follow up on interesting developments and to let the interviewees elaborate on various issues," as suggested by Dörnyei [21]. Berg [22] suggests that the advantage of a follow up interview is that it allows for "in depth probing while permitting the interviewer to keep the interview within the parameters traced out by the aim of the study. Hence, the follow up interviews on students' solution efforts allowed the researcher to uncover information about students' thoughts and challenges faced during their efforts to solve tasks on differential equations. Following Dörnyei [21] the researcher was able to rephrase or redirect questions that were not understood by the students during the follow up interviews.

\subsection{Validity and Reliability}

Issues of validity and reliability of research instruments were of great significance to this study as they contributed to the credibility of the research. Creswell [23] defines validity as the degree to which a study reflects the specific concepts it aims to investigate. Qualitative validity was of concern to this study whose focus is on the accuracy of the findings. Strategies employed in this study in pursuit of qualitative validity are triangulating data, use of member checking and use of peer debriefing.

First, following Corbin and Strauss' [24] suggestion, data were triangulated by comparing written responses and interview transcriptions of students' utterances from follow up interviews. Second, member checking validity strategy was employed whereby the researcher's interpretations of students' solution attempts were taken back to the students for their considerations. Member checking strategy afforded students the opportunity to comment on the findings on language related struggles experienced by the students when trying to resolve contextualized tasks on differential equations. Finally, the researcher applied peer debriefing to determine the accuracy of interpretations made. In this respect, a visiting lecturer on sabbatical leave examined the research report and posed some questions about how I determined the impact of language related challenges on students' learning of contextualized differential equations as suggested by Creswell [23].

Validity addresses the question; does research instrument allow the researcher to hit "the bull's eyes." Hence, measuring concepts involved in the study accurately would in turn allow the researcher to draw plausible conclusions in the sense of Cozby and Kuper [25]. In this research the task sheet contained two tasks on contextualized differential equations that the researcher thought would be appropriate for answering the research questions. To reduce researcher bias the researcher sought expertise consensus from the visiting lecturer who scrutinized data collection instruments. Another form of validity is external validity which tries to answer the question: can the findings be generalized? Cozby and Kuper 
[25] describe external validity as the extent to which the results can be generalized beyond the sample used in the study. The researcher reiterates that generalizability of findings was not the focus of this study that sought to develop a characterization of language related challenges in differential equations and how those challenges hinder students' learning of differential equations at high school level.

Reliability is the degree of consistency with which the instrument measures an attribute. Qualitative data collection techniques in the form written responses and follow up interviews allowed the researcher to generate thick descriptions to ensure reliability in the following manner. Data from written responses and follow up interviews were compared for consistency thereby serving as some reliability check as recommended by Lawson [26].

\subsection{Data Collection Procedures}

After developing data collection tools, the author of this article then sought permission from the District Schools Inspector to collect data from one urban high school in one of the provinces in Zimbabwe. First, the task sheet was administered in the classroom in which students worked individually on the tasks. No time restrictions were imposed on the respondents who worked on the tasks until complete solutions were generated or no further progress could be made. The researcher then scrutinized students' attempts for the purpose of determining responses which were not clear and required clarification from the students concerned. Second, after agreeing on the set of follow up questions with the visiting lecturer on sabbatical leave the author of this article then sought clarity on issues observed from the written responses. The follow up interviews were then audio-recorded. With regards to the setting of the interview, the researcher considered a number of issues. First, the researcher ensured that the interviews were conducted in a comfortable and private environment which was convenient for the respondents. Third, the researcher assured the mathematics students that the information they provided was going to be treated with utmost privacy and confidentiality. Finally, the researcher followed Strauss and Corbin's [24] suggestion that when working from a qualitative perspective, the researcher attempts to develop a holistic understanding of a phenomenon and data collection is shaped by preliminary data analysis as the investigation proceeds.

\subsection{Data Analysis}

In this qualitative research data analysis focused on developing an understanding of language related challenges on differential equations. Data analysis aimed at an increased understanding of language barriers to the learning of contextualized differential equations at Advanced level. First, the written responses were scrutinized to draw some sense of how language and mathematical notation used in word problems posed challenges to the students. Data analysis involved selecting from students' solution attempts those responses that typify the discordant translations between the objective figurative contexts and the students' construal of the problem contexts.

\section{Results and Discussion}

\subsection{Results and Discussion}

In this section the researcher presents and discusses findings pertaining to the research question: How do language related challenges experienced by students impede their learning of differential equations at Advanced level? Data from task sheets and follow up interviews were used to address this research question. Focus of the qualitative data analysis process was on teasing out discordant translations between students' solution efforts and the researchers' marking guides. Hence, from students' written responses and follow up interviews the researcher identified various sorts of language related difficulties experienced by students in their learning of contextualized differential equations. Typical students' responses are now presented and discussed.

Task 1

Fans arrive at the gate of soccer stadium at a rate inversely proportional to the time remaining before the gate is opened. The number of fans at the gate, $t$ minutes before it is opened is $x .10$ minutes before opening, there are 100 fans, and the rate of arrival is 50 per minute.

(a) Show that this situation can be modeled by differential equation $\frac{d x}{d t}=-\frac{500}{t}$

(b) Find: (i) The general solution of this equation

(ii) The number of fans present one minute before gate opens.

First, I present and discuss Gilbert's solution attempt to task 1.

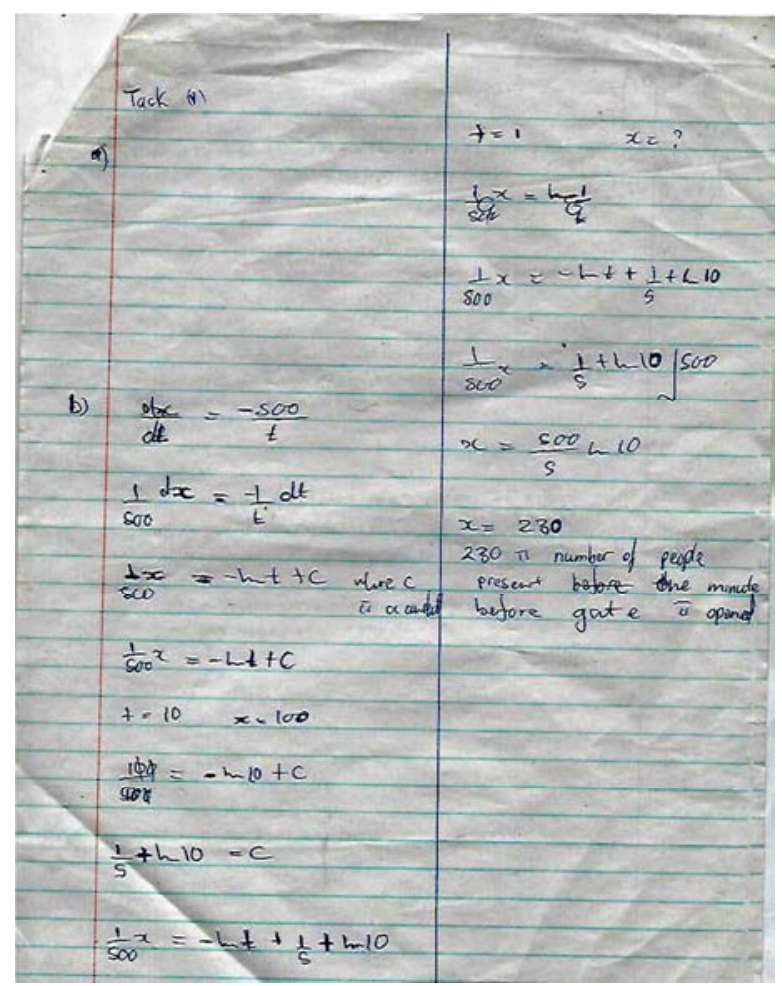

Figure 1. Gilbert's written response to task 1. 
Figure 1 reveals that Gilbert had a weak command of the subject content. He could not interpret the language in task 1 to derive the given differential equation as shown by the blank space in the solution attempt. High procedural fluency was demonstrated as Gilbert could separate variables and then proceeded to integrate the differential equation to come up with the general equation. He could also identify the initial conditions which were, when $x=100, t=10$ but he could not determine the exact value of the constant of integration. Consequently, Gilbert could not calculate the number of fans one minute before the gate was opened. Hence, while Gilbert was comfortable with procedural aspects of the differential equation he could not draw meaning from the narrative descriptions of the problem situation in the sense suggested by Lager [10].

Second, we consider Chris' solution attempts to task 1 .

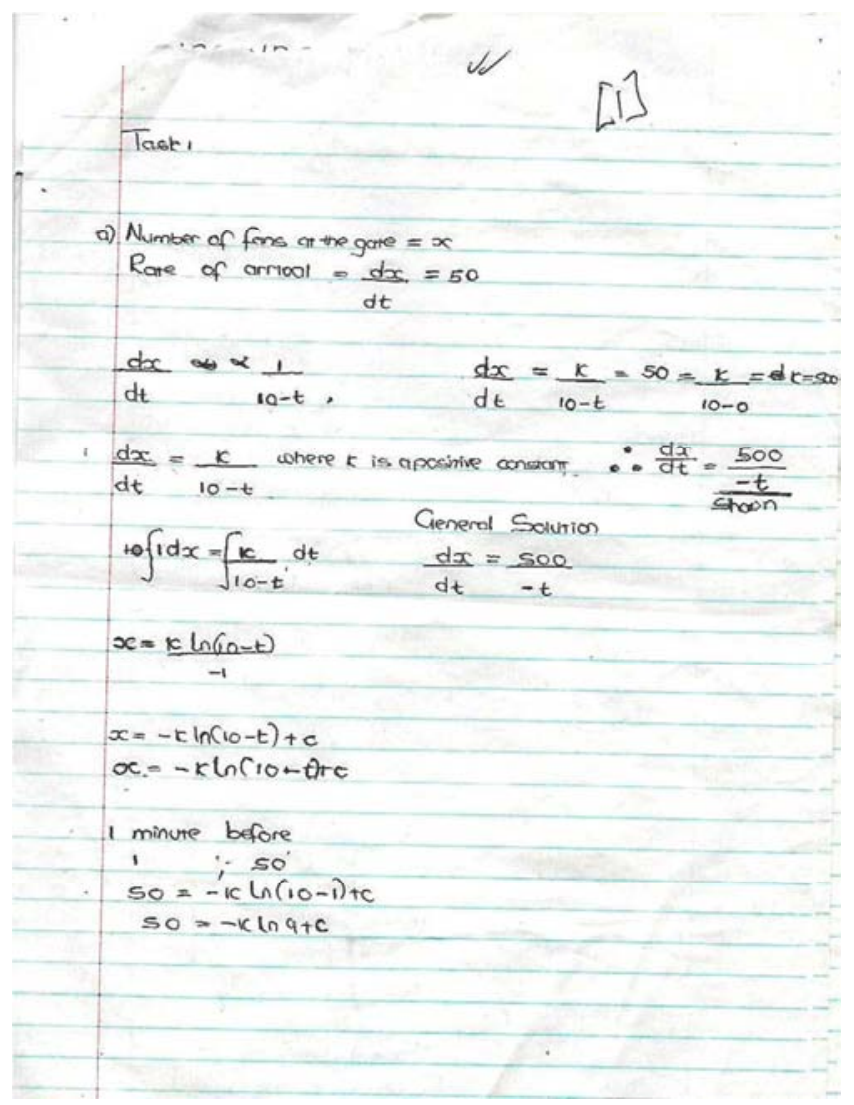

Figure 2. Chris'written response to task 1.

Chris demonstrated a strong command of procedural aspects in his solution attempt by succeeding in separating the variables and integrating the differential equation. However, the follow up interview revealed that he failed to explain how the negative sign involved in the derivation of differential equation comes into play. Chris' claim that, $\frac{d x}{d t} \propto \frac{1}{10-t}$, shows that he faced challenges in interpreting decreasing rate of change of quantities involved. Chris's effort parallels Gagnon and Abel's [18] argument that subtle differences in vocabulary such as those denoting relationships among ideas cause students to experience difficulties in learning mathematics. For instance, from the narration Fans arrive at the gate of soccer at a rate inversely proportional to the time remaining before the gate is opened, Chris was expected to infer that time was a decreasing quantity and hence a negative sign was appropriate. Chris failed to make such inferences and hence he could not formulate the required differential equation.

Third, we focus on Bether's effort to resolve task 1.

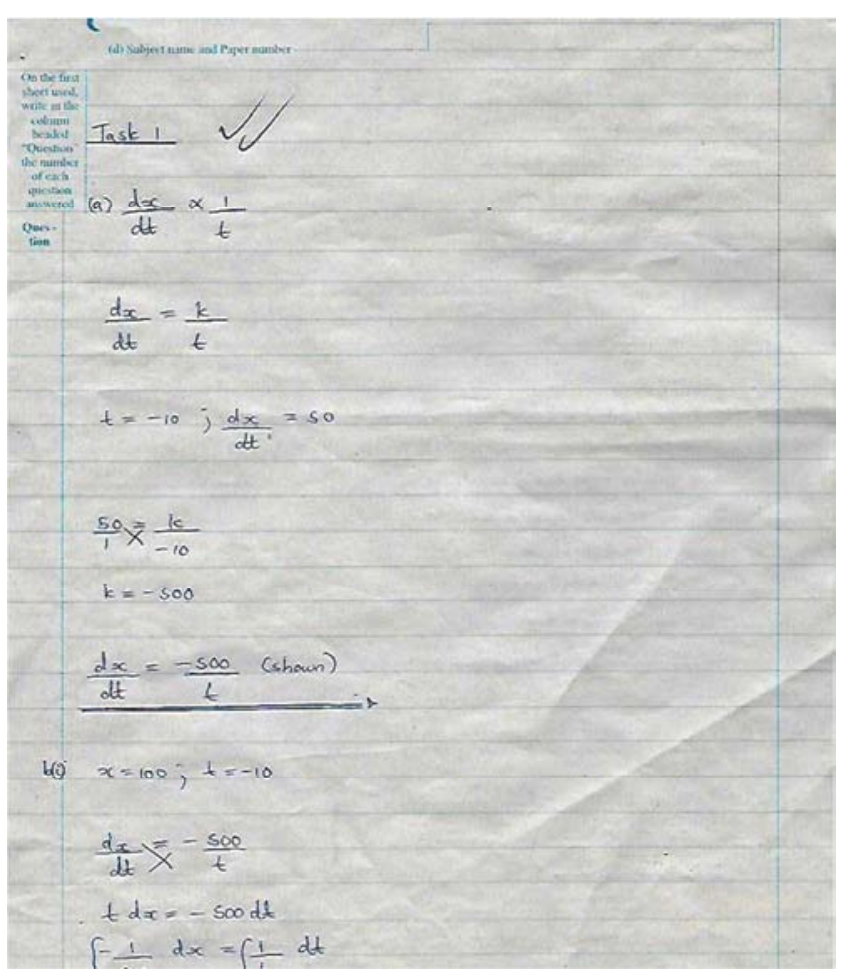

Figure 3. Extract of Bether's written response to task 1.

Figure 3 shows that Bether had no access to the technical language needed to derive the required differential equation. As illustrated in the extract of her solution attempt Bether had a fragile grasp of the initial conditions as her interpretation of remaining time led to the senseless formulation " $t=-10$," from the description of the problem she failed to apply the initial conditions to generate the particular solution. Hence, Bether's solution attempt reveals that similar to Chris's effort, Bether could not grasp the meaning of the negative sign from the information in task 1. During the follow up interview Bether claimed that the negative sign in the differential equation refers to a decrease in the rate of arrival of fans at the gate. This was wrong interpretation. In stark contrast, the rate of arrival of fans at the gate denoted an increase which was supposed to be accompanied by a corresponding decrease in time as we approached the time when the gate was going to be opened. Further, although Bether separated variables correctly, she failed to apply the initial conditions, $t=10, x=100$ to obtain a particular solution. As a result, she could not calculate the number of fans 1 minute before the gate was to be opened. It can be, therefore, inferred that Bether lacked adaptive reasoning as she could not think logically about relations among concepts pertinent to the task at hand, a finding that parallels results by Kilpatrick et al [11].

Finally, I focus on Nyarai's effort to tackle the task 1. 


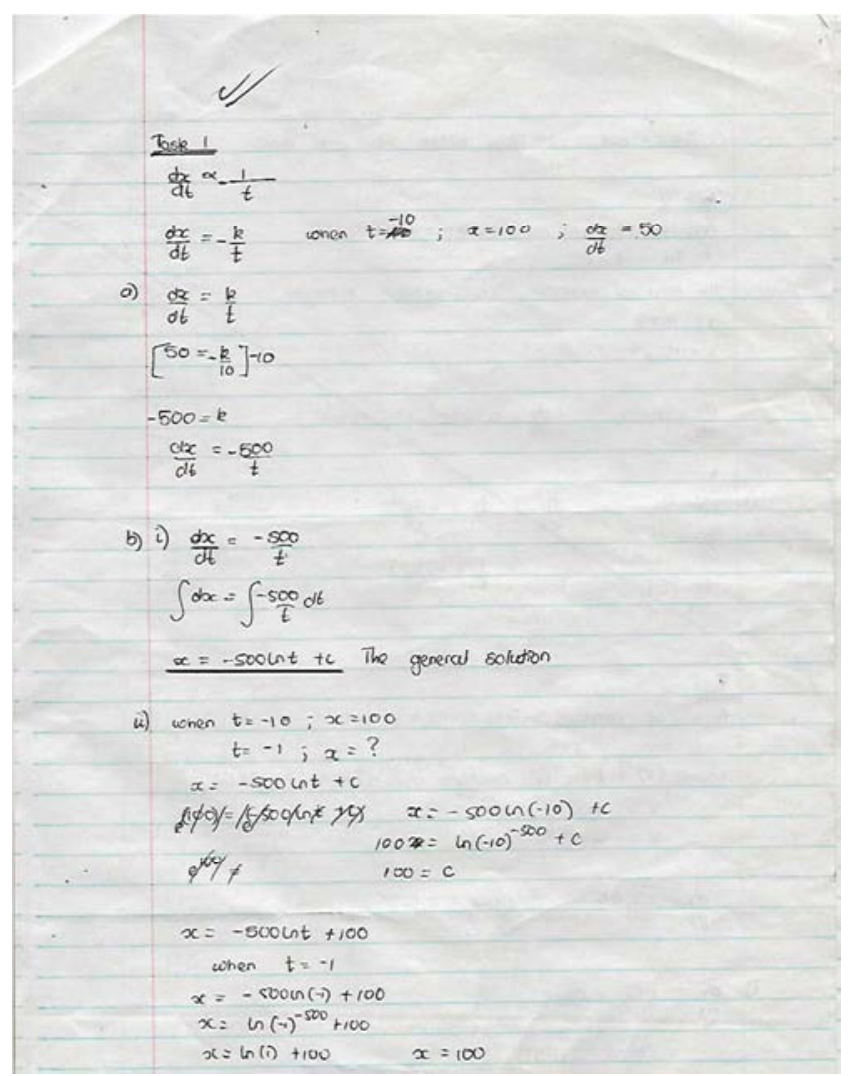

Figur 4. Extract of Nyarai's written response to task 1.

Similar to Bether, Figure 4 shows that Nyarai could also not extract meaning from the narrative description about the rate of arrival of soccer fans at the gate and as a result she could not formulate the differential equation required. For instance, no justification was provided as to how negative sign in $\frac{d x}{d t}=-\frac{k}{t}$ vanished for it to become $\frac{d x}{d t}=\frac{k}{t}$. Further, similar to Bether and Chris' efforts discussed earlier Nyarai also demonstrated a weak command of the given initial conditions. She wrote when " $t=-10$ " which points to the fact that Nyarai did not appreciate the essence that the assertion $t=-10 \mathrm{~s}$ is senseless. Nyarai could not draw meaning out of the initial conditions and hence she could not derive the differential equation. Nyarai's procedural fluency was strong as shown by successful separation of variables and correct application of integration of natural logarithms and she could determine the general solution. However, Nyarai's struggles with interpretation of given initial conditions hindered her progress she could not determine the number of fans present one minute before the gate was opened. For instance she wrote " $t=-1, x=$ ?" which led to the answer " $x=100$." From the description of Nyarai's attempt to task 1 , it can be seen that both her procedural and conceptual fluency with regards to the task were constrained by language related challenges. For instance, lack of grasp of vocabulary used such rate of arrival of fans being proportional to time remaining. These words should have influenced Nyarai to infer the sort of relationships in quantities involved namely, a decrease in time that should have been accompanied by an increase in the number of fans. Hence, similar to Gagnon and Abel's [18] results, Nyarai demonstrated lack of grasp of terms denoting relationships among concepts that caused her to experience difficulties in her efforts to solve tasks on differential equations.

Next, the researcher presents and discusses results of data analysis of students' solution attempts to task 2:

A water tank has the shape of a cuboid with base area $4 \mathrm{~m}^{2}$ and height $3 \mathrm{~m}$ and initially empty. Water is poured into the tank at a rate of $0.05 \mathrm{~m}^{3}$ per minute. There is a small hole at the bottom of the tank through which water leaks out. The depth of water in the tank is $h$ meters when the water has been poured in for t minutes.

In a simple, model it is assumed that water leaks out of the tank at a constant rate of $0.025 \mathrm{~m}^{3}$ per minute.

(i)) Show that the variable $h$ satisfies the differential equation $\frac{d h}{d t}=\frac{1}{160}$

(ii) Hence or otherwise, find the time when the tank starts to overflow.

In a more redefined model, then variable, $h$ satisfies the differential equation $160 \frac{d h}{d t}=2-h$.

(iii) Solve the differential equation, expressing $h$ in terms of $t$.

(iv) Hence sketch a graph of h in terms of $t$.

First, I consider Primrose's attempt to solve task 2.

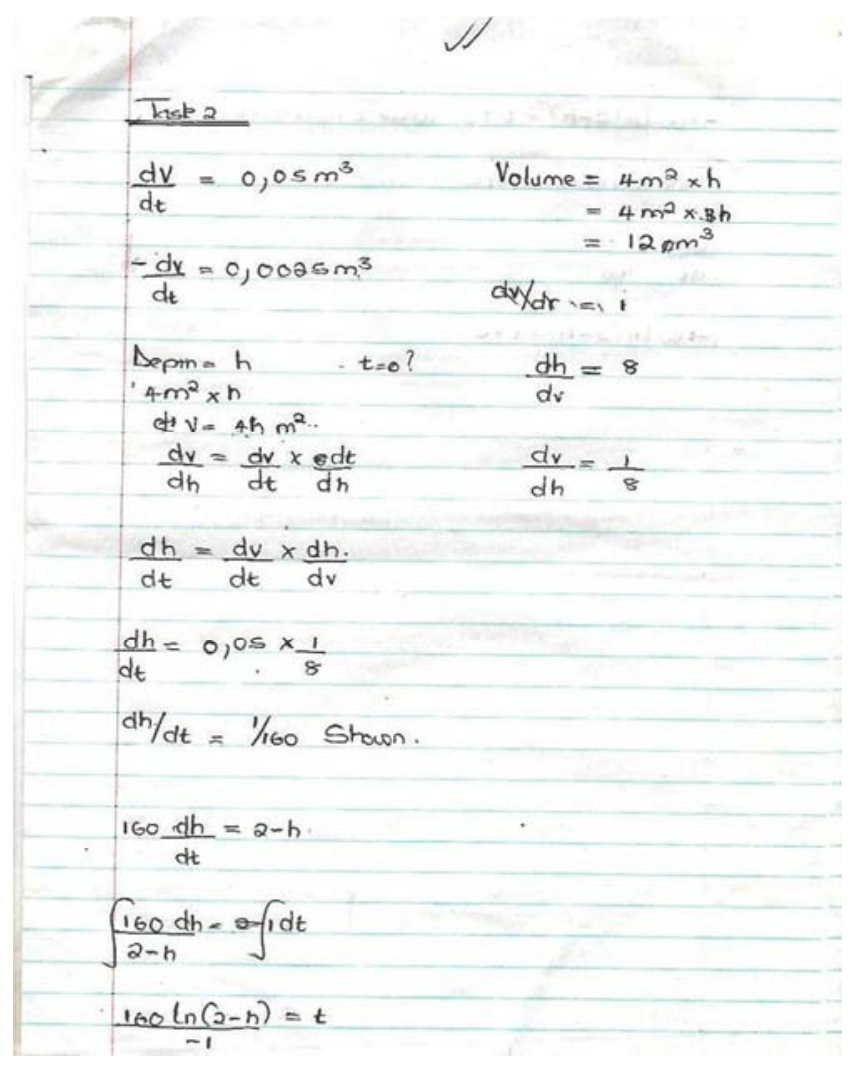

Figure 5. Primrose's written response to task 2.

Primrose's solution attempt reveals that she could not interpret the technical language in order to derive the required differential equation. For instance, her lack of grasp of verb-preposition collocation referring to an increase in the rate, $\frac{d h}{d t}$ negatively impacted on her attempts to derive the differential equation. Furthermore, Primrose could not use the 
chain rule $\frac{d h}{d t}=\frac{d v}{d t} \cdot \frac{d h}{d v}$. The claim by Primrose that "volume $=12 \mathrm{~m}^{3}$, " points to the fragility of her understanding of the idea that $V=4 h$. These limitations led to yet another false claim that $" \frac{d h}{d v}=8 "$ However, Primrose separated variables and went on to apply integration to determine the general solution but failure to access initial conditions led to an impasse after stating the general solution, $-160 \ln (2-h)=$ $t+c$. The student's solution attempt reveals high procedural fluency while her efforts to derive the differential equation showed low conceptual fluency (Riccomini et al., 2012). Primrose could not extract meaning from the narrative description of the initial conditions and so she could not determine the particular solution, that is, she could not compute time when the tank would start to overflow. Sketching of the solution curve could not be done contrary to the expectation that students at this level should be able to use multiple modes of representation of their knowledge of mathematics content - a result similar to Hewitt-Bradshaw's [16].

Second, Chris' solution attempt to the differential equation on task 2 is now examined. Similar to Primrose's attempt Chris could not also formulate the differential equation as shown in the following extract.

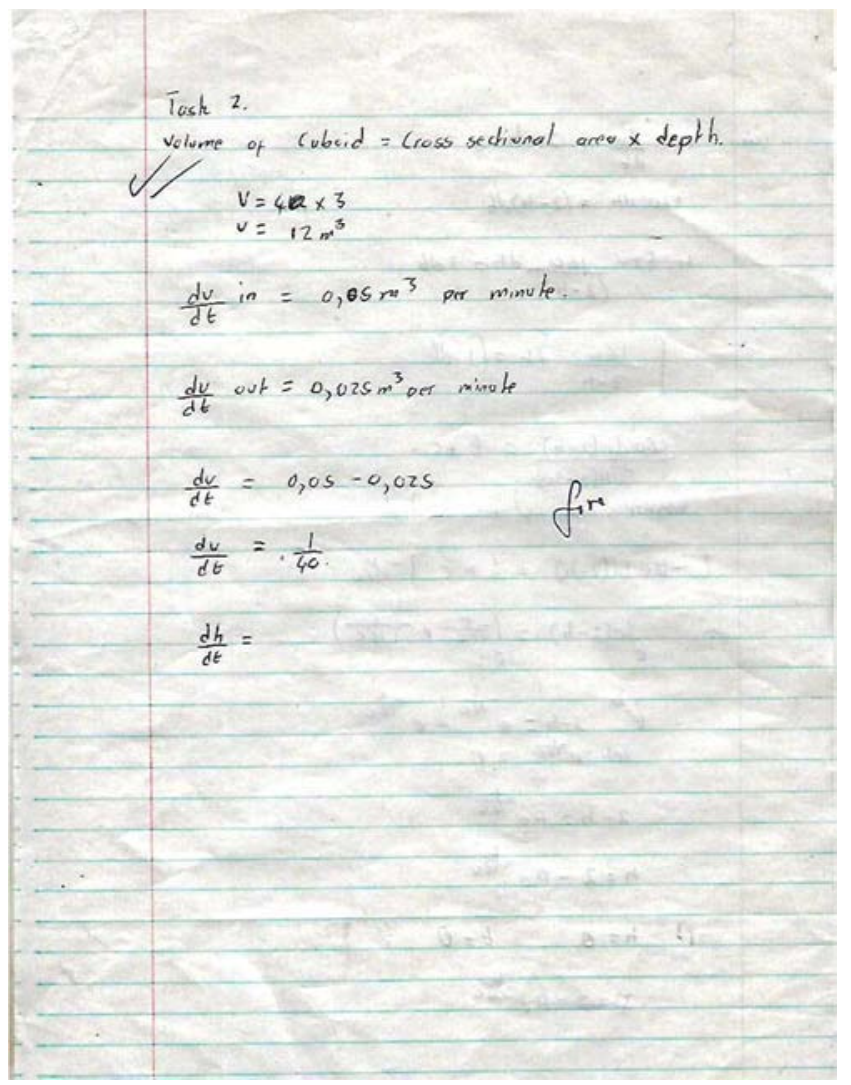

Figure 6. Extract of Chris'written attempt to task 2.

Figure 6 shows that while Chris demonstrated good grasp of verb preposition collocation " $\frac{d v}{d t} i n-\frac{d v}{d t} o u t$," used to denote the net rate of increase of volume of water in the tank with respect to time, Chris' could not access the chain rule $\frac{d h}{d t}=\frac{d v}{d t} \cdot \frac{d h}{d v}$. Hence, Chris could not proceed after noting that $\frac{d v}{d t}=\frac{1}{40}$. However, good procedural fluency was exhibited in item (iii) whereby Chris was able to separate variables and proceeded to integrate correctly to obtain the general solution " $160 \ln (2-h)=t+c$ " as shown in the next extract.

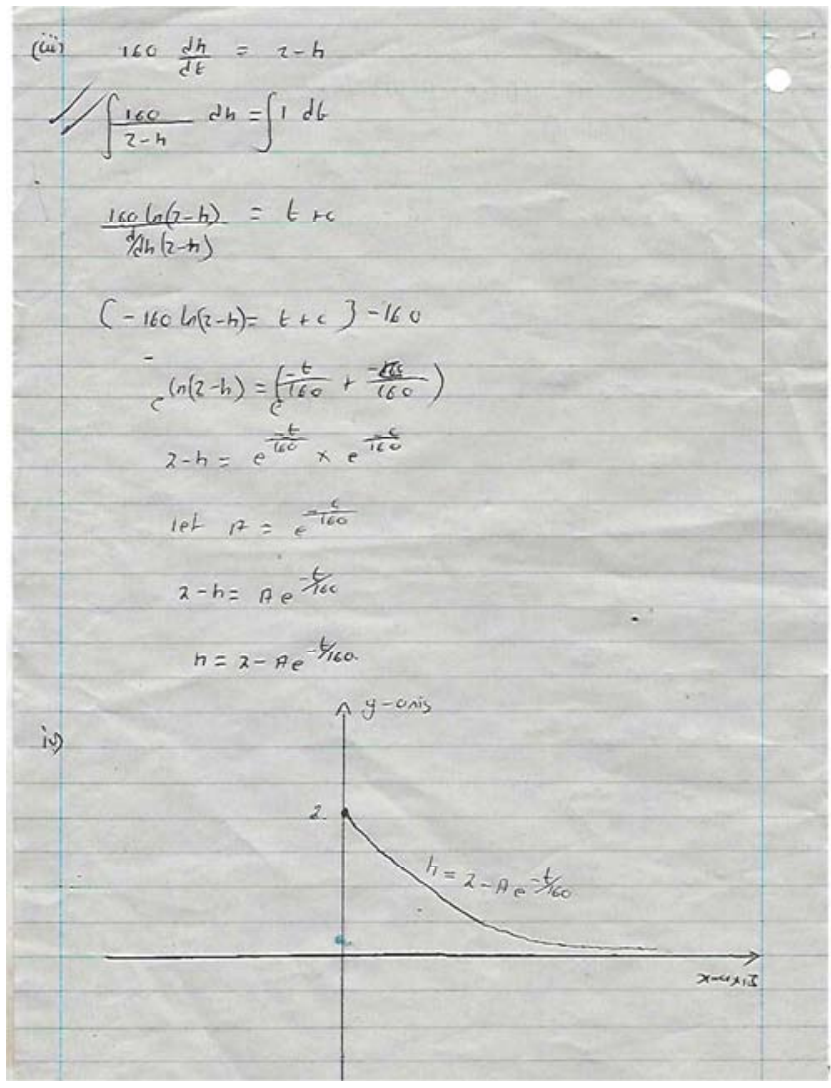

Figure 7. Extract of Chris'written response to task 2.

Figure 7 reveals that Chris could not extract meaning from given initial conditions $h=0, t=0$ which he should have inferred from the description that the tank was initially empty. Consequently, an awkward formulation " $h=2-A e^{-\frac{t}{160}}$ " of the solution was stated, an indication that he had a weak grasp of initial conditions of the problem. Furthermore, Chris' graphical instantiation revealed a complete mess in his chunk of reasoning with the differential equation task. For instance, besides being labeled with wrong $y$ and $x$ axes instead of $h$ (depth) and $t$ (time) the graph depicts an exponential decrease in the depth of water which is contrary to the expected rise in the depth of water owing to a net rate of increase of volume with time. Hence, Chris' woes were illuminated in his efforts to sketch the solution curve which was a futile attempt -an observation that parallels Hewitt-Bradshaw's [16] finding that learners experience serious discomforts when creating, representing and reasoning with visual representational forms of knowledge such as graphs.

Third, Nyarai's attempt to task 2 is presented next. 


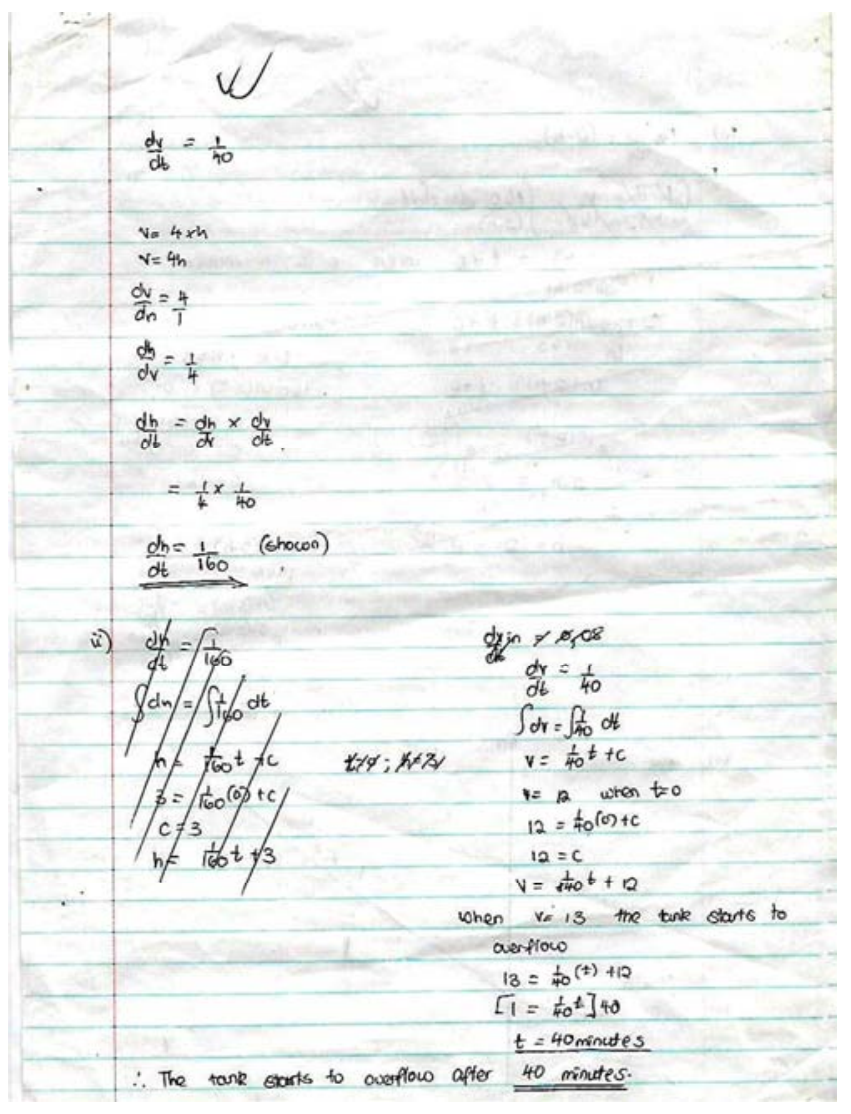

Figure 8. Nyarai's written response to task 2.

Nyarai's attempt to solve the differential equation reveals that she could interpret the verb-preposition collocation implied by a net rate of increase of water since the rate of inflow exceeded rate of leakage through a hole at the bottom of the tank as she wrote " $\frac{d v \text { in }}{d t}-\frac{d v \text { out }}{d t}=0,05-0,025$ " a problem solving behavior consistent with findings by Reccomini et al. [1]. Hence, she could derive the differential equation, $\frac{d h}{d t}=\frac{1}{160}$ by subsequently applying the chain rule. However, to determine when the tank would start to overflow the student resorted to the relation $\frac{d v}{d t}=\frac{1}{40}$. Nyarai then separated variables and integrated correctly to get $v=\frac{1}{40} t+$ $c$. Nyarai then claimed that " $v=12$ when $t=0$," - a false claim since it implies that the tank was initially full of water and yet the question specifies that the tank was initially empty. Nyarai's chunk of reasoning led to the false assertion that "the tank starts to overflow after 40 minutes." Hence, Nyarai's lack of grasp of vocabulary used to describe the initial conditions (tank initially empty): $t=0, h=0$, led to her failure to generate the particular solution that could then be used to calculate the time, $t$ when $h=3$.

The researcher now focuses on Nyarai's attempt to the item involving the refined model of the rate of flow of water into the tank.

Figure 9 shows that good procedural fluency was exhibited by Nyarai as she could separate variables and integrate the differential equation to get the solution " $-160 \ln (2-h)=$ $t+c$." However, her woes with the differential equation persisted when she used wrong initial conditions " $t=0 ; h=$
3." Furthermore, her unsuccessful attempt to sketch the graph of the particular solution of the differential equation were a surprising observation since the researcher had anticipated that the student would use different semiotic codes to represent her knowledge of contextualized differential equations. Hence, language related challenges experienced in trying to extract meaning from the narrative descriptions of given initial conditions accounted for unsuccessful solution attempts by Nyarai.

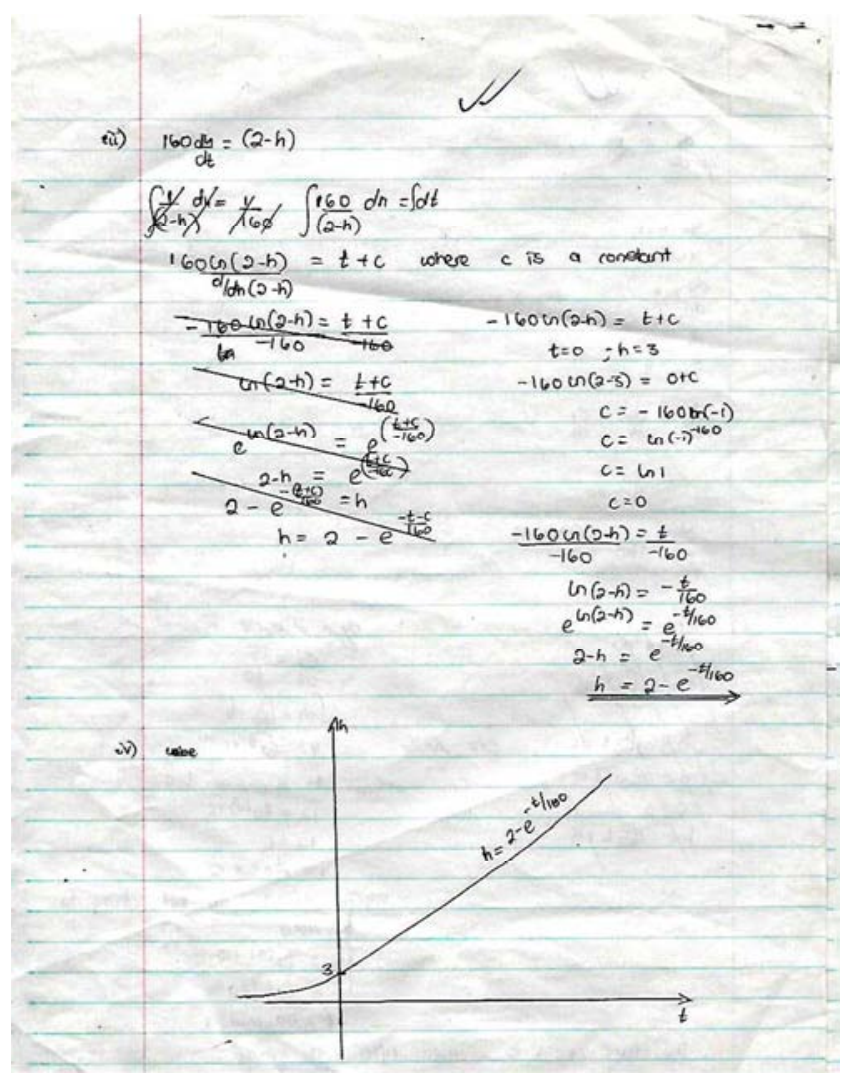

Figure 9. Extract of Nyarai s solution attempt to task 2.

Fourth, Grace' attempt to the first part of task 2 is now examined. Grace realized that she was supposed to use the chain rule, $\frac{d h}{d t}=\frac{d h}{d v} x \frac{d v}{d t}$, and she proceeded to derive the differential equation by noting that $\frac{d v}{d t}=0.025$ and $\frac{d v}{d h}=4$. After succeeding in separating the variables, Grace proceeded to integrate the differential equation to obtain the general solution and could use the initial conditions $t=0, h=0$ to obtain the particular solution. Furthermore, Grace made the correct interpretation $h=3 \mathrm{~m}$ in order to determine when the tank starts to overflow. It can be inferred that the student could use different semiotic codes to represent her knowledge of the contextualized differential equation by switching from one form of representation to another as was also reported by Hewitt-Bradshaw [16]. For instance, Grace could establish connection between the particular solution and the general solution by strategically applying the given initial conditions to determine the time when the tank starts to overflow, that is, when $h=3 m$. Next, we focus on Grace's written response to item (ii) of task 2. 


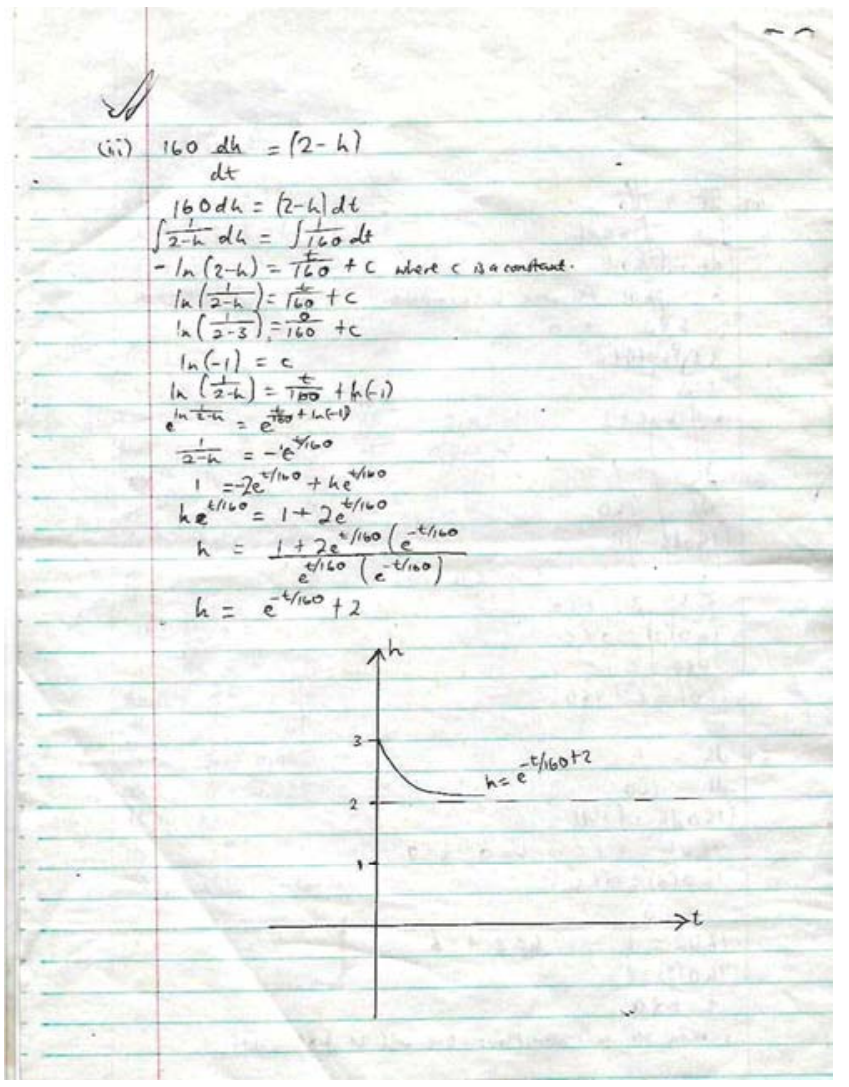

Figure 10. Grace's written response to task 2.

With respect to the item involving the refined model, Grace demonstrated low conceptual fluency by failing to apply the same initial conditions $t=0, h=0$ to determine the particular solution from the general solution " $-\ln (2-h)=\frac{1}{160} t+$ c." Hence, despite the fact she had earlier on shown good procedural fluency by being able to integrate correctly, use of $h=3 m, t=0$ as initial conditions revealed lack of desired interplay between her procedural and conceptual knowledge. This led to a false pictorial overview of the refined model shown by the graph. The graphical illustration gives the impression that the tank was initially full - a false assertion that is in stark contrast to the description of objective figurative context that " $A$ water tank has the shape of a cuboid with base area $4 m^{2}$ and height $3 m$ and initially empty."

Finally, Bether's written response to items (i) and (ii) of task 2 are now presented and discussed.

Figure 11 reveals that Bether could interpret the technical language involved in order to derive the differential equation. Lager [10] asserts that grasp of technical vocabulary is essential for successful problem solvers in in mathematics. The extract shows that Bether was able to access the technical vocabulary and the chain rule because Bether could derive the differential equation which was then successfully solved to get the solution $t=160 h$. Furthermore, Bether went on to determine the time when the tank could start to overflow which was somewhat a form of evidence that the student had a good conceptual fluency of the objective figurative context of the differential equation. The researcher reiterates the fact that having access to relevant technical language affords students some access to mathematical concepts and relationships among those concepts. Bether's solution attempt to item (ii) of task 2 is now presented.

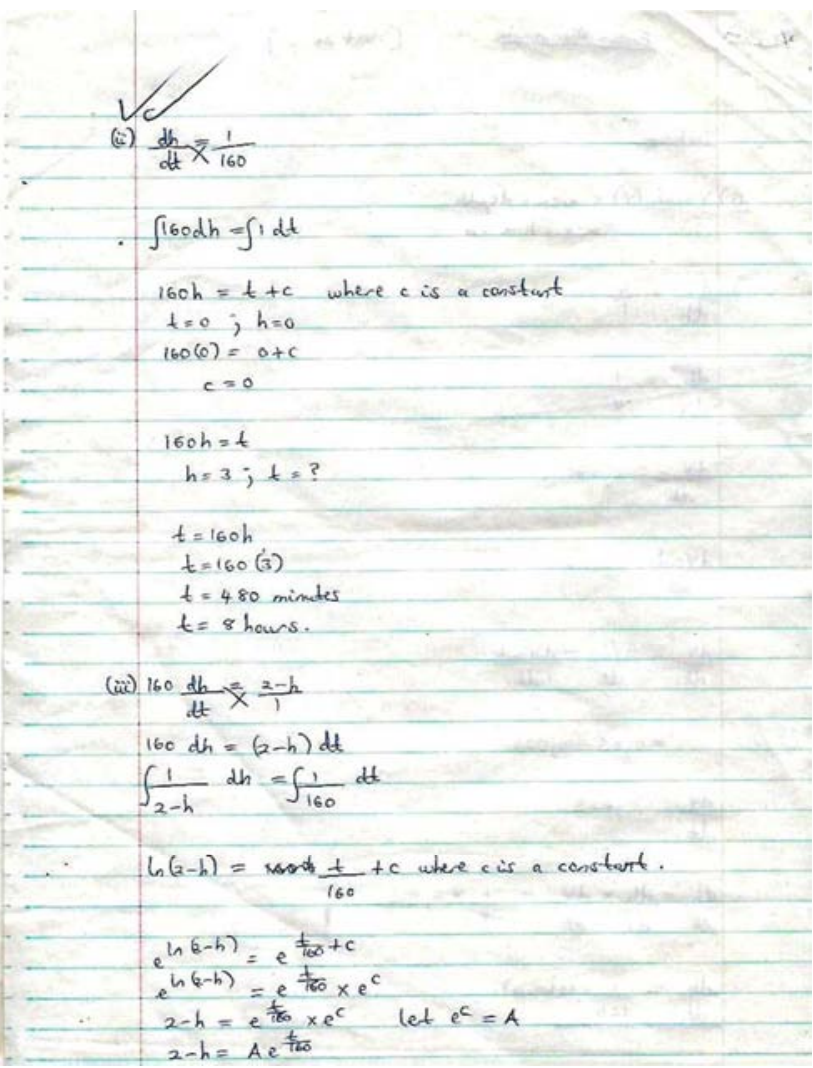

Figure 11. Extract of Bether's written response to task 2.

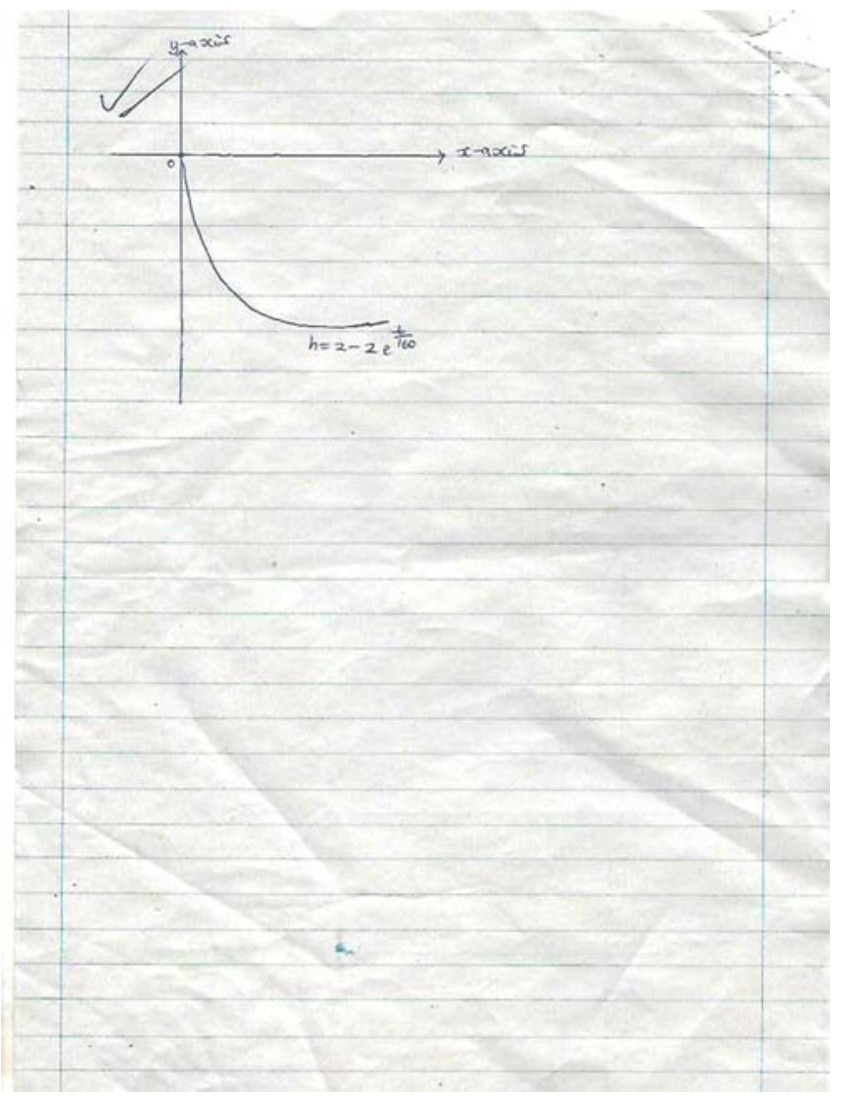

Figure 12. Extract of Bether's solution attempt to task 2. 
With regards to the item involving the refined model of flow of water into the tank, Figure 11 reveals that although Bether separated variables correctly she made an error when integrating the differential equation that led to the solution " $h=2-2 e^{\frac{t}{160}}$. " An extract of Bether's graphical representation of her solution attempt is now presented.

The graphical representation of the solution shown illumines the student's language related barriers as Bether did not relate her pictorial to the narrative description of the problem. For instance the assertion that "the tank starts to overflow" pointed to the fact the height increases with time contrary to what the graph depicts. Hence, Bether's effort exhibited lack of the desired coordination between her procedural and conceptual fluency in the sense of Riccomini et al [1]. Hence, the student did not reflect on the essence of solution process in relation to objective figurative context of the differential equation.

\subsection{Conclusion and Implications for Practice}

From our discussion of results pertaining the research question, How do language related difficulties experienced Advanced level students interfere with their the learning of differential equations?, the following conclusions were drawn.

First, the researcher concluded that students had severe challenges in extracting meaning from the objective figurative contexts of the differential equations pointing to an overall low conceptual fluency. Second, it was also concluded that despite exhibiting high procedural fluency when solving differential equations, the study has uncovered a weak coordination between students' procedural and conceptual knowledge that can be attributed to language related challenges such as students' struggles with interpretation of given initial conditions of contextualized differential equations. These are serious limitations in Advanced level students' knowledge structures which deserve the attention of research mathematicians and mathematics educators in order to improve the learning of differential equations and related topics.

\section{Acknowledgements}

I express my sincere appreciation and thanks to the visiting lecturer Professor E. Chinamasa for his encouragement, Ms G Sunzuma, a mathematics educator for revising the manuscript and making useful suggestions. Finally, I thank my former undergraduate mathematics education student, Greatwin Jaji for her assistance during data collection.

\section{References}

[1] P. J. Riccomini, G. W. Smith, E. M. Hughes, K. M. Fries, The language of teaching and learning mathematical vocabulary, Reading and Writing Quarterly, 31(3) (2015), 235-252.

[2] A. Lemke, (2003). Standardized achievement tests and English language learners, Psychometric issues and Educational Assessment, 8 (2003), 231-257.
[3] Van Rinsveld, C. Schiltz, M. Brunner, K. Landerl and S. Ugen, Solving Arithmetic Problem in First and Second Language Context, Learning and Instruction, 42 (2016), 72-82.

[4] M. K. Sarabi and K. Abdul Gafoor, Influence of Linguistic Challenges on Attitudes Towards Mathematics Learning among Upper Primary Students of Kerala, Proceedings of International Seminar on Priorities, Barriers and Directions to Education, 2015, 1-9.

[5] D. Cummis, W. Kintsch, K. Reusser and R Weimer, The role of understanding and solving word problems, Cognitive Psychology, 20(200), 405-438.

[6] M. Martiniello, Linguistic complexity, thematic representations, and differential item functioning for English language learners with maths tests, Educational Assessment, 14 (2009), 160-179.

[7] K. Lesseig, Investigating mathematical knowledge for teaching proof in professional development, International Journal of Research in Education and Science, 2(2) (2016), 253-270.

[8] D. Clarke and S. Helme, (1996). Context as construction, Proceedings of CIEAM 47, Berlin, (1996), 379-389.

[9] Z. Ndemo and D. J. Mtetwa, Preferences of contexts for learning mathematics expressed by students in rural secondary school environments of Zimbabwe, Zimbabwe Journal of Research, 22(1) (2010), 1-15.

[10] C. Lager, Types of mathematics-language reading interactions that unnecessarily hinder algebra learning and assessment, Reading Psychology, 27(2-3) (2006), 163-204.

[11] J. Kilpatrick, J. Swafford and B. Findell, Adding it up: Helping children learn mathematics. Washington, DC: National Academy Press, 2001.

[12] G. Boulet, How does language impact the learning of mathematics? Let me count the ways, Journal of Teaching and Learning, 5(1) (2007), 1-12.

[13] A. Selden and J. Selden, Validations of Proofs considered as texts: Can Undergraduates Tell an Argument Proves a Theorem? Journal for Research in Mathematics Education, 34 (2003), 4-36.

[14] E. C. Mulwa, The Role of Language in Mathematics in Students' Understanding of Number Concepts in Eldoret Municipality, Kenya, International Journal of Humanities and Social Sciences, 4(3) (2014), 264-274.

[15] J. Zwiers, The third language of academic English, Educational Leadership, 62(4) (2005), 60-63.

[16] L. P. Hewitt-Bradshaw, Language issues in mathematics and science: An analysis of Caribbean reports on students' performance in Caribbean Secondary Education Certificate Examinations, Caribbean Curriculum, 19(2012), 43-66.

[17] N. Fatmanissa and K. Kusnandi, The Linguistic Challenges of Mathematics Word Problems: A Research and Literature Review, Malaysian Journal of Learning and Instruction, Special Issue on Graduate Students Research on Education, 73-92.

[18] M. J. Gagnon and S. K. Abel, ELLS and Language of school science, Science and Children, 46(5) (2009), 50-51. 
[19] J. Huang, B. Normandia and S Greer, Communicating mathematically: Comparison of knowledge structures in teacher and student discourse in a secondary mathematics classroom, Communication Education, 54(1) (2005), 34-51.

[20] B. Durrheiim, G. MacMillan and W. Schumacher, Designing Qualitative Research (4th ed). Thousand Oaks, CA: Sage, 2004.

[21] Z. Dörnyei, Research Methods in Applied Linguistics: Quantitative Qualitative, and Mixed Methodologies, Oxford University Press, 2007.

[22] B. L. Berg, Qualitative research methods for the social sciences, London Pearson, 2007.
[23] J. W. Cresswell, Research Design: Qualitative, Quantitative, and Mixed Methods Approaches, Thousand Oaks, CA: Sage, 2014.

[24] J. Corbin and A. Strauss,(2008). Basics of qualitative research, Thousand Oaks, Sage, 2008.

[25] E. Cozby and A. Kuper, Critically appraising qualitative research. BMJ, 337 (a) (2009), 1035.

[26] T. Lawson, Cambridge sociology: an interview with Tony Lawson, Erasmus Journal of Philosophy and Economics, 2(1) (2009), 100-122. 